\title{
The Clinical Research Office of the Endourological Society Percutaneous Nephrolithotomy Global Study: Nephrolithotomy in 189 Patients with Solitary Kidneys
}

\author{
Viorel Bucuras, M.D., Ganesh Gopalakrishnam, M.D., J. Stuart Wolf Jr., M.D., ${ }^{3}$ \\ Yinghao Sun, M.D., Giampaolo Bianchi, M.D., ${ }^{5}$ Tibet Erdogru, M.D., \\ and Jean de la Rosette, M.D., ${ }^{7}$ on behalf of the CROES PCNL Study Group
}

\begin{abstract}
Background and Purpose: The study compared characteristics and outcomes in patients with solitary and bilateral kidneys who were treated with percutaneous nephrolithotomy (PCNL) in the Clinical Research Office of the Endourological Society (CROES) PCNL Global Study.

Patients and Methods: Data from consecutively treated patients from 96 centers worldwide were collated after a 1-year period. The following variables in patients undergoing PCNL with solitary or bilateral kidneys were compared: Prevalence, patient characteristics, intraoperative differences and outcomes, including bleeding and transfusion rates, renal function, and stone-free rates.

Results: Data from 5803 patients were collated; 189 (3.3\%) with solitary and 5556 (96.7\%) with bilateral kidneys. Patient characteristics were well matched generally with the exception of cardiovascular disease and American Society of Anesthesiologists (ASA) risk scores, which were significantly greater in patients with solitary than with bilateral kidneys $(P<0.0001$ and $P=0.004$, respectively). Patients with solitary kidneys had also undergone significantly more procedures to remove calculi before this survey than bilateral patients $(P=00.049-<0.0001)$. Levels of renal impairment were significantly greater $(P<0.0001)$ and stone-free rates were significantly lower $(P=0.001)$ post-PCNL in solitary than bilateral kidney patients. Although bleeding rates were the same in both groups, transfusion rates were significantly greater in solitary kidney patients $(P=0.014)$.

Conclusions: Patients with a solitary kidney had a higher cardiovascular risk and ASA score. Outcomes related to morbidity and stone-free rate were less favorable for solitary kidneys.
\end{abstract}

\section{Introduction}

$\mathbf{R}$ ENAL AGENESIS AND RENAL DYSPLASIA ARE commonly occurring renal congenital anomalies that produce the condition of solitary kidney. While the prevalence of congenital solitary kidney is unknown because it is often asymptomatic, it is estimated by autopsy that 1 in 1000 persons may have been born with just a single kidney, although ultrasonographic scanning suggests this frequency may be higher at 1 in 500 of the general population. ${ }^{1,2}$ Congenital malformations, however, are not the sole cause. Unilateral nephrectomy after trauma, cancer or nephrolithiasis or sec- ondary to the complications of nephrolithiasis and its treatment also contributes to the number of persons with solitary kidneys as does increasing kidney donation. ${ }^{2}$ Subsequently, solitary kidneys may be more common than currently estimated.

The presence of kidney stones in persons with bilateral kidneys is known to have detrimental effects and, if not promptly treated, may cause renal insufficiency and eventually renal failure. ${ }^{3}$ The frequency of renal insufficiency in patients with kidney stones is highly variable, but in one study, $6.3 \%$ of patients had a serum creatinine level of $>1.5 \mathrm{mg} / \mathrm{dL}$, and rates of renal insufficiency between $0.78 \%$ to

\footnotetext{
${ }^{1}$ Department of Urology, Timisoara Clinical Emergency Hospital, “Victor Babes" University of Medicine and Pharmacy, Timisoara, Romania.

${ }^{2}$ Department of Urology, Vedanayagam Hospital, RS Puram, India.

${ }^{3}$ Department of Urology, University of Michigan Medical Center, Ann Arbor, Michigan

${ }^{4}$ Department of Urology, Changhai Hospital, Shanghai, China.

${ }^{5}$ Department of Urology, University of Modena \& Reggio Emilia, Modena, Italy.

${ }^{6}$ Department of Urology, Akdeniz University Hospital, Antalya, Turkey.

${ }^{7}$ Department of Urology, AMC University Hospital, Amsterdam, The Netherlands.
} 
$17.5 \%$ have been reported from a variety of other centers. ${ }^{3}$ Furthermore, duration of nephrolithiasis, its recurrence, and its treatment with multiple procedures also has a negative effect on renal function. ${ }^{4}$

While it was known that stone removal was beneficial, before the introduction of percutaneous nephrolithotomy (PCNL), concerns were expressed about the effects of the procedure on kidney function and associated complications, particularly in patients with solitary kidneys. The technique of PCNL has been practiced for more than 30 years and has constantly undergone refinements. It is currently the preferred first-line therapy for renal stones that are not amenable to extracorporeal shockwave lithotripsy (SWL) or ureteroscopy (URS), such as large volume and staghorn calculi. ${ }^{5}$ PCNL is not known to cause significant functional nephron damage, and many patients with renal impairment experience improvement in renal function post-PCNL. ${ }^{6-8}$ Nevertheless, the management of calculi with PCNL remains challenging, particularly in those patients with solitary kidneys.

The Clinical Research Office of the Endourological Society (CROES) instigated the PCNL Global Study to generate a global database on the utility of the technique as assessed primarily by stone-free rate 30 days post-PCNL, but also through assessment of morbidity and other factors influencing outcomes. ${ }^{9}$ The current analysis compares outcomes in patients who present with solitary or bilateral kidneys.

\section{Patients and Methods}

The CROES PNCL Global Study organization and methods have been described in detail elsewhere. ${ }^{10}$ The study is a prospective observational study of consecutive patients who were treated at each participating center over the course of a single year. Overall, data were gathered from 96 centers worldwide. Patients who were eligible for inclusion had undergone PCNL for primary or secondary treatment of kidney stones; there were no specific exclusion criteria. Local guidelines and practices for PCNL procedures were followed.

Ultrasonography and/or radiography in combination with fluoroscopy guided upper tract access. Thereafter, and after caliceal puncture of the collecting system, a guidewire was inserted and maneuvered toward the ureter. Dilation of the tract to allow positioning of an access sheath and passage of a rigid nephroscope was attained with either balloon, telescopic, or serial dilators. After nephroscopic inspection, complete stones were removed using graspers or, if needed, lithotripsy by laser, ultrasound, or ballistic means was undertaken to fragment larger stones. The procedure was considered complete when all removable stones had been extracted. Stone-free status was determined at 30 days using a choice of radiography at the discretion of the treating physician. Bleeding severity was judged by the treating physician, and transfusions were administered according to local practice guidelines.

Within the database of patients who were treated with PCNL, we identified those with a solitary kidney, identified on the basis of upper tract imaging studies including ultrasonography, intravenous urography, or cross-sectional imaging.

\section{Data analysis}

Patients were classified depending on the presence or absence of anomalies into those with solitary or bilateral kidneys. Data on a number of variables, including patient characteristics, intraoperative differences, including puncture site, access location, method of guidance and dilation, and outcomes, including bleeding rates, operative time, pre- and postoperative serum creatinine concentrations, were analyzed to compare differences between groups.

SPSS version 16.0 was used to analyze the collected data. All data are descriptive and based on frequencies. Continuous variables were analyzed using the Mann-Whitney $U$ test while the Pearson chi-square test was used for comparison of categorical variables.

\section{Results}

A total of 5803 patients were included in the CROES database during the period between November 2007 and December 2009. Of these, 58 patients were excluded from the analysis, because information on whether they had solitary kidneys was not recorded. Of the remaining 5745 patients, 189 $(3.3 \%)$ had solitary and $5556(96.7 \%)$ had bilateral kidneys.

\section{Patient characteristics}

Patient characteristics according to kidney status are shown in Table 1. Data on origin of the solitary kidney were unknown and might have been from congenital, traumatic, oncologic, nonfunctional, or donation causes. The proportion of males to females in each group was broadly similar. The mean age of those with solitary kidneys was, however, slightly higher, although not significantly so. Both groups had similar proportions of patients with diabetes, and median body mass index (BMI) was also well matched. A significantly greater proportion of patients with solitary kidneys had cardiovascular disease, however, as reflected in the significantly greater proportion with American Society of Anesthesiologists (ASA) scores of 2 to $4(P=0.004)$. A greater proportion of patients with solitary kidneys used anticoagulants and corticosteroids, although statistically significant differences between groups in use of these drugs were not noted.

Considering previous procedures, patients with solitary kidneys had undergone PCNL $(P=0.049)$, pyelolithotomy, and nephrostomy $(P<0.0001)$ significantly more frequently than those patients with bilateral kidneys; there were no significant differences in proportions of patients having undergone previous SWL or URS. No significant differences in median stone burden, positive preoperative urine cultures, or in the proportion of patients with staghorn stones were observed between groups.

\section{Intraoperative procedures}

Details of the intraoperative procedures used, including guidance and dilation techniques, and access and renal puncture sites in each of the patients groups, are shown in Table 2. Overall, no statistically significant differences between groups were observed. Generally, patients in both groups had a greater proportion of lower and middle punctures than upper kidney punctures, although a slightly greater proportion of patients with solitary kidneys than with bilateral kidneys had middle punctures and slightly more bilateral kidney patients experienced lower renal punctures. Cutaneous access in both groups was most frequently made below the 12th rib. The ratio of balloon to telescopic/serial dilation was broadly similar between groups. 
Table 1. Patient Characteristics

\begin{tabular}{|c|c|c|c|}
\hline Patient characteristic & Solitary kidney & Bilateral kidneys & $\mathrm{P}$ value \\
\hline Sex (male/female) & $58.7 / 41.3$ & $56.4 / 43.6$ & 0.568 \\
\hline Mean (SD) age (years) & $51.6(15)$ & $49.1(16)$ & 0.055 \\
\hline Median (IQR) BMI & $27.0(24-30)$ & $26.7(23-29)$ & 0.227 \\
\hline \multicolumn{4}{|l|}{ Proportion of patients, N (\%) } \\
\hline With diabetes & $24(12.8 \%)$ & $748(13.5 \%)$ & 0.872 \\
\hline Using anticoagulants & $14(7.5 \%)$ & $300(5.4 \%)$ & 0.289 \\
\hline Using prednisolone & $5(2.7 \%)$ & $64(1.2 \%)$ & 0.126 \\
\hline With CVD & $66(35.1 \%)$ & $1262(22.8 \%)$ & $<0.0001$ \\
\hline Positive urine culture & $34(18.6 \%)$ & $847(15.8 \%)$ & 0.364 \\
\hline With staghorn stones & $46(26.4 \%)$ & $1404(27.4 \%)$ & 0.855 \\
\hline \multicolumn{4}{|c|}{ Proportion of patients having undergone previous procedures, N (\%) } \\
\hline PCNL & $37(19.6 \%)$ & $781(14.2 \%)$ & 0.049 \\
\hline SWL & $40(21.3 \%)$ & $1172(21.2 \%)$ & 0.990 \\
\hline Pyelolithotomy & $33(17.7 \%)$ & $429(7.8 \%)$ & $<0.0001$ \\
\hline URS & $22(11.8 \%)$ & $536(9.7 \%)$ & 0.425 \\
\hline Nephrostomy & $46(24.5 \%)$ & $388(7.1 \%)$ & $<0.0001$ \\
\hline \multicolumn{3}{|c|}{$\begin{array}{l}\text { Proportion of patients with systemic disease assessed } \\
\text { according to ASA assessment scale }(\%)\end{array}$} & 0.004 \\
\hline ASA grade 1 & $76(41.8 \%)$ & $2929(53.6 \%)$ & \\
\hline ASA grade 2 & $74(40.7 \%)$ & $1909(34.9 \%)$ & \\
\hline ASA grade 3 & $28(15.4 \%)$ & $587(10.7 \%)$ & \\
\hline ASA grade 4 & $4(2.2 \%)$ & $47(0.9 \%)$ & \\
\hline Median (IQR) stone burden $\left(\mathrm{mm}^{2}\right)$ & $347.00(110-630)$ & $314.00(130-550)$ & 0.110 \\
\hline
\end{tabular}

$\mathrm{SD}=$ standard deviation; $\mathrm{IQR}=$ interquartile range; $\mathrm{BMI}=$ body mass index; $\mathrm{CVD}=$ cardiovascular disease; $\mathrm{PCNL}=$ percutaneous nephrolithotomy; SWL = shockwave lithotripsy; URS= ureteroscopy; ASA = American Society of Anesthesiologists.

\section{Outcomes}

The principal postoperative outcomes are shown in Table 3. Stone-free rates were significantly lower $(P=0.001)$ and the need for blood transfusion was significantly higher $(P=0.014)$ in patients with solitary kidneys compared with those with bilateral kidneys. Mean preoperative and first day postoperative serum creatinine concentrations were significantly higher in the solitary kidney group $(P<0.0001$; Fig. 1$)$; in both groups, serum creatinine concentrations were elevated on the

TAble 2. Intraoperative Characteristics

\begin{tabular}{lccc}
\hline $\begin{array}{l}\text { Intraoperative } \\
\text { characteristic }\end{array}$ & $\begin{array}{c}\text { Solitary } \\
\text { kidney }\end{array}$ & $\begin{array}{c}\text { Bilateral } \\
\text { kidneys }\end{array}$ & $\begin{array}{c}\mathrm{P} \\
\text { value }\end{array}$ \\
\hline Puncture site (\%) & $17(9.0 \%)$ & $556(10.1 \%)$ & 0.079 \\
$\quad$ Upper & $41(21.7 \%)$ & $874(15.8 \%)$ & \\
$\quad$ Middle & $111(58.7 \%)$ & $3653(66.2 \%)$ & \\
Lower & $20(10.6 \%)$ & $439(8.0 \%)$ & \\
Multiple & $4(2.1 \%)$ & $80(1.5 \%)$ & 0.757 \\
Location of access (\%) & $27(14.4 \%)$ & $829(15.3 \%)$ & \\
Above 11th rib & $156(83.4 \%)$ & $4526(83.3 \%)$ & \\
Above 12th rib & $21(11.2 \%)$ & $567(10.4 \%)$ & \\
$\quad$ Below 12th rib & $121(64.7 \%)$ & $3455(63.6 \%)$ & \\
Guidance (\%) & $25(13.4 \%)$ & $819(15.0 \%)$ & \\
US & $20(10.7 \%)$ & $591(10.9 \%)$ & \\
Fluoroscopy & $72(39.1 \%)$ & $2174(40.9 \%)$ & \\
$\quad \begin{array}{l}\text { Fluoroscopy + US } \\
\text { Other }\end{array}$ & $112(60.9 \%)$ & $3135(59.1 \%)$ & \\
Dilation method (\%) & & & \\
$\quad$ Balloon & & \\
$\quad$ Telescopic dilators & & & \\
\hline
\end{tabular}

$\mathrm{US}=$ ultrasonography. first day postoperatively compared with preoperative values. Similar results were reported in both groups of patients concerning percentage of failed procedures and the occurrence of hydrothorax, perforation, and fever.

\section{Discussion}

As part of an ongoing series of CROES PCNL Global Study analyses, the article presented here compares outcomes in patients with solitary vs bilateral kidneys. The 3.3\% rate of PCNL procedures in patients with solitary kidneys identified in the CROES database might be thought to be relatively low until the estimated occurrence of solitary kidney within the general population is considered. If the frequency of solitary kidney as estimated by ultrasonography is 1 in 500, this equates to a population frequency of congenital solitary kidney of approximately $0.002 \% .^{2}$ Even taking into consideration the likely incidence of solitary kidney from other causes, the frequency of solitary kidney noted in this survey suggests that kidney stones are diagnosed or occur more frequently in patients with solitary kidneys than in patients with bilateral kidneys.

That kidney stones are being diagnosed more frequently in patients with solitary kidneys (possibly through more diligent monitoring by the physician) is supported by the observation of the statistically significantly increased occurrence of previous PCNL, pyelolithotomy, and nephrostomy procedures noted in patients with solitary compared with bilateral kidneys. Clearly, it is important to remove obstructions and reinstate drainage of urine promptly in patients with solitary kidney to prevent further injury to a sole functioning kidney. Whether a greater proportion of patients with solitary kidneys experience more severe renal blockages associated with 
Table 3. Outcome after Percutaneous Nephrolithotomy

\begin{tabular}{lccr}
\hline Outcome measure & Mono kidney & Bilateral kidneys & P value \\
\hline Median (IQR) operative time (min) & $75.0(55-120)$ & $75.0(50-110)$ & 0.173 \\
No. (\%) stone free & $123(65.4 \%)$ & $4189(76.1 \%)$ & 0.001 \\
Re-treatment methods & $1 / 31(3.2 \%)$ & $79 / 842(9.3 \%)$ & \\
No. (\%) URS & $16 / 31(51.6 \%)$ & $372 / 842(44.2 \%)$ & \\
No. (\%) PCNL & $11 / 31(35.4 \%)$ & $342 / 842(40.6 \%)$ & \\
No. (\%) SWL & $3 / 31(9.6 \%)$ & $48 / 842(5.7 \%)$ & 0.280 \\
No. (\%) Other & $19(10.2 \%)$ & $424(7.7 \%)$ & 0.014 \\
No. (\%) bleeding & $19(10.1 \%)$ & $306(5.6 \%)$ & 0.997 \\
No. (\%) blood transfusions & $5(6.9 \%)$ & $148(7.0 \%)$ & $<0.0001$ \\
No. (\%) blood transfusions among & $14(12.6 \%)$ & $114(4.6 \%)$ & $<0.0001$ \\
$\quad$ balloon dilated & $1.4(0.74)$ & $1.0(0.43)$ & $<0.0001$ \\
No. (\%) blood transfusions among & $1.5(0.8)$ & $1.1(0.43)$ & 0.242 \\
$\quad$ telescopic dilated & $25(13.3 \%)$ & $570(10.4 \%)$ & 0.573 \\
Mean (SD) preoperative serum $\quad$ rreatinine concentration (mg/dL) & $8(4.3 \%)$ & $180(3.3 \%)$ & 0.434 \\
Mean (SD) first day postoperative & $2(1.1 \%)$ & $102(1.9 \%)$ & 0.471 \\
$\quad$ serum creatinine concentration (mg/dL) & $2(1.1 \%)$ & $97(1.8 \%)$ & \\
No. (\%) fever & & & \\
No. (\%) perforations & No. (\%) hydrothorax & & \\
No. (\%) failed procedures & & & \\
\hline
\end{tabular}

$\mathrm{IQR}=$ interquartile range; URS=ureteroscopy; $\mathrm{PCNL}=$ percutaneous nephrolithotomy; $\mathrm{SWL}=$ shockwave lithotripsy; $\mathrm{SD}=$ standard deviation.

kidney stones remains questionable, however, because no significant difference in the frequency of staghorn stones was noted between groups.

No statistically significant difference in the ratio of males to females was noted between groups. It is believed that congenital renal agenesis affects approximately twice as many males as females. ${ }^{1}$ If this is the case, and if the majority of patients who were identified by CROES have congenital renal

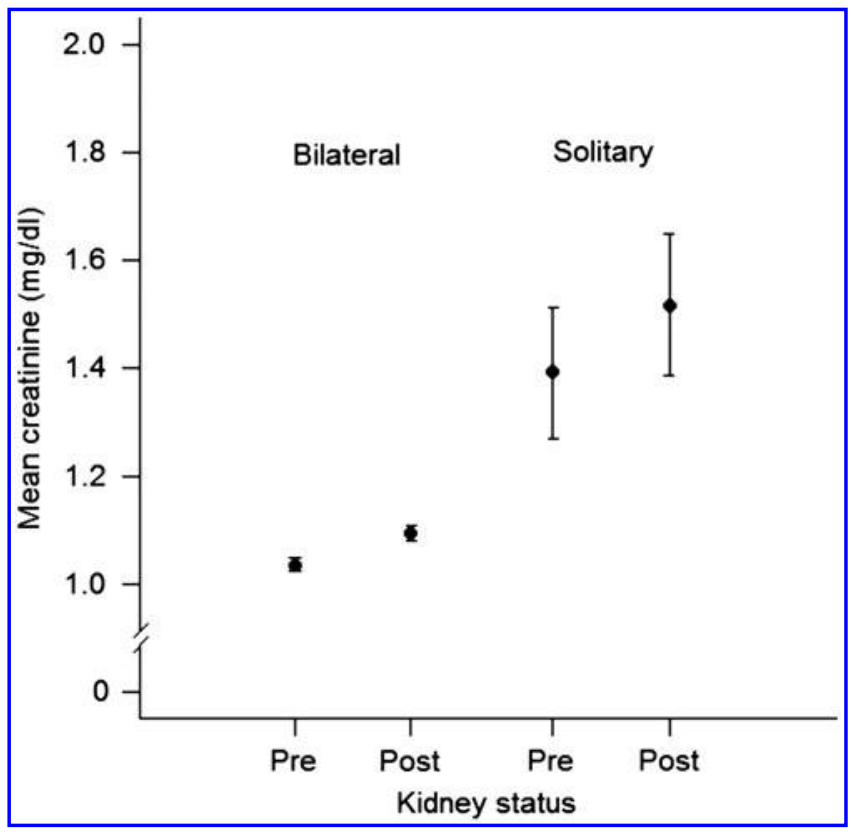

FIG. 1. Serum creatinine levels pre- and postpercutaneous nephrolithotomy in patients with a solitary kidney or bilateral kidneys. agenesis, one might have expected the ratio of males to females in the solitary kidneys group to be skewed toward a much greater proportion of affected males than that seen. Such was not the case, however, suggesting that other reasons, such as a greater loss of kidneys to trauma or cancer occurs more frequently in females, may "balance" the gender ratio. Without knowing the underlying origins of solitary kidneys in our cohort, however, such suggestions must remain speculative.

A greater proportion of patients with solitary kidneys than bilateral kidneys had cardiovascular disease (CVD). There was no difference, however, in the frequency of diabetes or in median BMI between the groups, suggesting that the increased frequency of CVD noted in the solitary kidneys group was because of more than the usual cardiovascular risk factors. Similarly, ASA risk scores were also greater in patients with solitary than bilateral kidneys, suggesting that solitary kidney patients are more likely to have serious systemic disease.

Are patients with solitary kidneys at higher risk of systemic and CVD than those with bilateral kidneys because of their functional renal impairment? It has been observed that over time, kidney function decreases in children with a solitary, functional kidney. ${ }^{11}$ This may be despite, or possible because of the mechanisms, such as glomerular hypertrophy, that often come into play to compensate for loss of renal mass. Serum creatinine levels are raised compared with controls, indicating that renal function is impaired. ${ }^{12}$ The level of renal impairment, however, may be slight and can remain stable for many years; only in later life might serum creatinine levels indicative of true renal impairment become apparent.

With regard to outcomes, in the current analysis of the PCNL Global Study, significant differences in median preoperative and pos-operative serum creatinine concentrations were noted between groups. That serum creatinine levels were higher and, therefore, that renal function was impaired 
in those patients with solitary kidneys was not unexpected. Renal compensatory hypertrophy was likely the cause of this observation, although it should be noted that the median preoperative value of serum creatinine in the solitary kidney patients is indicative of mild to moderately impaired renal function. That mean serum creatinine values in both patient groups were elevated on the first postoperative day and therefore that renal function was further slightly impaired was also to be expected and has been observed elsewhere. ${ }^{13,14}$ In these reports, serum creatinine levels returned to normal within 2 weeks and, while data were not available, it is anticipated that this would also be the case in the patients reported here.

Bleeding during and after PCNL continues to be a cause of patient morbidity. While no significant differences in the occurrence of bleeding was noted between groups, the need for transfusion was almost doubled in solitary kidney compared with bilateral kidney patients. One explanation for this could be that slightly more patients with solitary kidneys were using anticoagulants and, therefore, the bleeding might be more difficult to staunch. Hypertrophy of the kidney is also a recognized risk for excessive bleeding, which could further contribute to the increased transfusion requirement in solitary kidney patients. ${ }^{15}$ More importantly, the condition of a higher ASA score and CVD may be an important factor to decide, even at lower threshold, for blood transfusion. Also stone size, upper caliceal puncture, multiple punctures, staghorn stones, and inexperienced surgeon are risk factors for hemorrhage post-PCNL. ${ }^{15,16}$ Because PNCL is the most complicated stone treatment to teach, the importance of the risk associated with the inexperienced surgeon in PCNL should never be underestimated. ${ }^{17}$

Stone removal in solitary kidney patients needs to strike a balance between aggressive clearance and safety, and it may be that the balance reflected in our cohort is not yet optimal. The significantly lower stone-free rate noted in solitary compared with bilateral kidneys patients suggests that urologists were being cautious in the approaches being taken with stone removal, possibly resulting in less rigorous removal of stone fragments to avoid bleeding and any damage that might compromise renal function. In PCNL, surgical bleeding is the main cause of blood loss. Optimizing renal access, tract dilation, and renal manipulation while minimizing technical error in addition to an awareness of stone size during extraction, minimal nephroscope angulation, and the exertion of minimal torque forces on the kidney during PCNL all serve to reduce intraoperative bleeding and therefore the need for transfusion. ${ }^{18}$

Nevertheless, excessive risk reduction may come at the expense of an optimal stone-free rate, and clearly a balance must be struck between more aggressive stone clearance, thereby reducing the need for future interventions an optimizing renal function, and excessive caution in managing patients with solitary kidneys. Patients with solitary kidneys, particularly those with more impaired renal function, should be treated as any other patients. ${ }^{7}$ The findings of recent studies in which stone-free rates of $93.7 \%$ can be attained and in which outcomes and complication rates after PCNL were comparable in identically treated solitary kidney patients and appropriately matched controls suggests that just such a balance can be struck. ${ }^{3,7,19}$

This study was limited by several factors: First, the data obtained were not homogenous because the centers had dif- ferent methods to assess stone-free rates and operative times. This may potentially have resulted in lack of a unified reporting of outcomes. Furthermore, the biochemical makeup of the stones was not captured in the database. This limited our ability to compare outcomes based on the chemical compositions of the stones treated in the two groups. Finally, the database did not fully capture the cause of the solitary kidney status.

\section{Conclusion}

A higher cardiovascular risk and ASA score were seen in patients with a solitary kidney. Outcomes that were related to morbidity and stone-free rate were less favorable for patients with solitary kidneys.

\section{Acknowledgment}

The Global CROES PNCL study was supported by an unrestricted educational grant from Olympus.

\section{Disclosure Statement}

No competing financial interests exist.

\section{References}

1. Shapiro E, Goldfarb DA, Ritchey ML. The congenital and acquired solitary kidney. Rev Urol 2003;5:2-8.

2. Woolf AS, Hillman KA. Unilateral renal agenesis and the congenital solitary functioning kidney: Developmental, genetic and clinical perspectives. BJU Int 2007;99:17-21.

3. Yaycioglu O, Egilmez T, Gul U, et al. Percutaneous nephrolithotomy in patients with normal versus impaired renal function. Urol Res 2007;35:101-105.

4. Marangella M, Bruno M, Cosseddu D, et al. Prevalence of chronic renal insufficiency in the course of idiopathic recurrent calcium stone disease: Risk factors and patterns of progression. Nephron 1990;54:302-306.

5. Soucy F, Ko R, Duvdevani $M$, et al. Percutaneous nephrolithotomy for staghorn calculi: A single center's experience over 15 years. J Endourol 2009;23:1669-1673.

6. Chandhoke PS, Albala DM, Clayman RV. Long-term comparison of renal function in patients with solitary kidneys and/or moderate renal insufficiency undergoing extracorporeal shock wave lithotripsy or percutaneous nephrolithomy. J Urol 1992;147:1226-1230.

7. Goel MC, Ahlawat R, Kumar M, Kapoor R. Chronic renal failure and nephrolithiasis in a solitary kidney: Role of intervention. J Urol 1997;157:1574-1577.

8. Jones DJ, Kellett MJ, Wickham JE. Percutaneous nephrolithotomy and the solitary kidney. J Urol 1991;145:477-480.

9. The Clinical Research Office of the Endourological Society (CROES). http://www.croesoffice.org/ONGOINGPROJECTS/ PCNLstudy/tabid/63/Default.aspx.

10. de la Rosette J, Assimos D, Desai M, et al. The Clinical Research Office of the Endourological Society Percutaneous Nephrolithotomy Global Study: Indications, complications, and outcomes in 5803 patients. J Endourol 2011;25:11-17.

11. Vu KH, Van Dyck M, Daniels H, Proesmans W. Renal outcome of children with one functioning kidney from birth. A study of 99 patients and a review of the literature. Eur J Pediatr 2008;167:885-890.

12. Dursun H, Bayazit AK, Cengiz N, et al. Ambulatory blood pressure monitoring and renal functions in children with a solitary kidney. Pediatr Nephrol 2007;22:559-564. 
13. Canes D, Hegarty NJ, Kamoi K, et al. Functional outcomes following percutaneous surgery in solitary kidney. I Urol 2009;181:154-160.

14. Ekelund L, Lindstedt E, Lundquist SB, et al. Studies on renal damage from percutaneous nephrolitholapaxy. I Urol 1986;135:682-685.

15. El-Nahas AR, Shokeir AA, El-Assmy AM, et al. Postpercutaneous nephrolithotomy extensive hemorrhage: A study of risk factors. J Urol 2007; 177:576-579.

16. Srivastava A, Singh KJ, Suri A, et al. Vascular complications after percutaneous nephrolithotomy: Are there any predictive factors? Urology 2005;66:38-40.

17. de la Rosette JJ, Laguna MP, Rassweiler JJ, Conort P. Training in percutaneous nephrolithotomy-a critical review. Eur Urol 2008;54:994-1001.

18. Lee KL, Stoller ML. Minimizing and managing bleeding after percutaneous nephrolithotomy. Curr Opin Urol 2007; $17: 120-124$.

19. Resorlu B, Kara C, Oguz U, et al. Percutaneous nephrolithotomy for complex caliceal and staghorn stones in patients with solitary kidney. Urol Res 2011;39:171-176.
Address correspondence to: Jean J.M.C.H. de la Rosette, M.D.

Department of Urology AMC University Hospital Maibergdreef 9

1105 AZ Amsterdam Z-O The Netherlands

E-mail: J.J.delaRosette@amc.uva.nl

DOI: 10.1089/end.2012.0058

\title{
Editorial Comment for Bucuras et al.
}

\author{
Nicole. L. Miller, M.D.
}

$\mathbf{T}$ HE PRIMARY OBJECTIVE of any research study is to answer some important question. As researchers, we are commonly limited to reporting on our own experience, but haven't we all wished for more? More patients, more centers, just plain more data with the hope that the old saying, "There is power in numbers" rings true and provides us with the answers we have been seeking. This is the foundation on which the Clinical Research Office of the Endourological Society (CROES) was created. The CROES initiative "aims to promote and support high quality international patientcentered research in a transparent way and to facilitate the implementation of research projects by creating a global network." ${ }^{1}$ The CROES mission relies on worldwide collaboration and its stated vision is clear: "To apply rigorous scientific evaluation to the field of endourology with the intent to bring patients globally the most effective and efficient care possible." The CROES Percutaneous Nephrolithotomy (PCNL) study was the first to test the feasibility of a project this ambitious. It is as a part of this larger PCNL study that this report on patients with solitary kidneys was conducted.

While there is no question that the efforts of the CROES PCNL study group should be applauded, there are inherent challenges to a project of this magnitude that can impact the conclusions gathered from the data. I think we can categorize these strengths and limitations into those that relate to study size and those that result from study design.
CROES data are collected in a prospective manner through a remote data management system (DMS). The CROES PCNL study collects data from 96 centers in 26 countries and includes 5803 patients. The power of a study this size is immediately evident when you consider the clinical entity described in this article. Solitary kidney is uncommon enough that single institutions would be limited to small numbers of patients and be hard-pressed to draw any significant conclusions, but the CROES PCNL Global study includes 189 patients worldwide. In fact, this article suggests that outcomes related to morbidity and stone-free rate were less favorable for the solitary kidneys. The logistical challenge of collecting data from nearly 100 separate centers, however, makes it such that CROES is reliant on self-reporting and data entry into the DMS. This type of self-reported data can impact the quality of the data gathered by introducing the potential for missing key data points, unreliable data, infrequent data entry, etc. Furthermore, some countries have stricter Institutional Review Board (IRB) regulations than others; therefore, the ability to include all cases in a prospective manner for 1 year will be different in each center, because the centers that require IRB approval and patient consent for inclusion will be unlikely to have every patient consent to participate. Items that are even more difficult to control include the heterogeneity of the surgical selection, the approach, and the 
This article has been cited by:

1. Gokhan Atis, Cenk Gurbuz, Ozgur Arikan, Mert Kilic, Sabri Pelit, Cengiz Canakci, Samet Gungor, Turhan Caskurlu. 2013. Retrograde Intrarenal Surgery for the Treatment of Renal Stones in Patients With a Solitary Kidney. Urology . [CrossRef]

2. 2012. Journal of Endourology 26:12, 1535-1539. [Citation] [Full Text HTML] [Full Text PDF] [Full Text PDF with Links]

3. 2012. Journal of Endourology 26:10, 1253-1256. [Citation] [Full Text HTML] [Full Text PDF] [Full Text PDF with Links]

4. Vishwajeet Singh, Rahul Janak Sinha, Manoj Kumar, Dheeraj Kumar Gupta, Deeapak Nagathan, Arvind Gupta. 2012. Percutaneous Nephrolithotomy in Solitary Kidneys with or without Renal Failure: Does Nadir Serum Creatinine Predict LongTerm Renal Function?. UroToday International Journal 05:06. . [CrossRef] 